\title{
For Spacious Skies: Self-Separation with “Autonomous Flight Rules” in US Domestic Airspace
}

\author{
David J. Wing ${ }^{1}$ \\ NASA Langley Research Center, Hampton, VA, 23681 \\ William B. Cotton ${ }^{2}$ \\ National Institute of Aerospace, Hampton, VA, 23666
}

\begin{abstract}
Autonomous Flight Rules (AFR) are proposed as a new set of operating regulations in which aircraft navigate on tracks of their choice while self-separating from traffic and weather. AFR would exist alongside Instrument and Visual Flight Rules (IFR and VFR) as one of three available flight options for any appropriately trained and qualified operator with the necessary certified equipment. Historically, ground-based separation services evolved by necessity as aircraft began operating in the clouds and were unable to see each other. Today, technologies for global precision navigation, emerging airborne surveillance, and onboard computing enable traffic conflict management to be fully integrated with navigation procedures onboard the aircraft. By self-separating, aircraft can operate with more flexibility and fewer flight restrictions than are required when using ground-based separation. The AFR concept proposes a practical means in which self-separating aircraft could share the same airspace as IFR and VFR aircraft without disrupting the ongoing processes of Air Traffic Control. The paper discusses the context and motivation for implementing self-separation in US domestic airspace. It presents a historical perspective on separation, the proposed way forward in AFR, the rationale behind mixed operations, and the expected benefits of AFR for the airspace user community.
\end{abstract}

\section{Introduction}

$\mathrm{T}$ HOSE words from "America the Beautiful" have resonated with many generations of Americans who have marveled at the vastness of our country. It is also a matter of great national pride that powered flight was first achieved here, as well as the means for exploiting aviation's enormous potential for commerce, transportation, recreation, and national security. Safety has always been a primary consideration, not only in the evolution of aircraft design, but in the creation of operating practices to enable the beneficial uses of flying while preventing aircraft accidents that so often result in loss of life and property. But as the processes for ensuring the safety of flying try to keep up with the increasing numbers and variety of flight operations, the skies no longer seem so spacious, and restrictions to flight begin to impinge upon the economic and practical viability of the very operations they are designed to protect. Fortunately, great advances in processing and sensing technologies enable us to build upon the impressive capabilities embodied in our National Airspace System, and can unlock aviation's potential to use our vast airspace. The operating concept presented here embodies those technologies and provides new rules and procedures to help keep the US on aviation's leading edge, preserving the safety and utility of flight while protecting its ability to grow and prosper into the future, "for spacious skies".

This paper discusses the context and motivation for implementing self-separation in US domestic airspace. The proposed operational concept is titled Autonomous Flight Rules (AFR). Section II presents a historical view of navigation and separation. Section III introduces AFR and discusses what it means and takes to self-separate. Section IV addresses the operators intended for AFR, how AFR mixes with existing Instrument Flight Rules (IFR) and Visual Flight Rules (VFR) operations, and why creating a new set of flight rules is the prudent way forward. Section V presents anticipated user benefits, and Section VI summarizes and concludes the paper.

\footnotetext{
${ }^{1}$ Research Engineer, Mail Stop 152, david.wing@nasa.gov, AIAA Member.

${ }^{2}$ Aviation Consultant, 106 Snipe Ct, Lakeway, TX, wmcotton@gmail.com.
}

1

American Institute of Aeronautics and Astronautics 
A detailed description of the AFR concept is presented in Ref. 1. It is strongly recommended as companion reading to this paper for a more in-depth understanding of the concept's details than is possible to relate here.

\section{Historical Perspective}

\section{A. Visual and Procedural Separation}

The earliest aviators enjoyed complete operational freedom in an environment devoid of rules. But they were limited by their physical capabilities to fly only in "visual" conditions without the flexibility to operate in adverse weather or in clouds. Before gyro instruments, it was not possible to maintain control of the airplane without a visible horizon, let alone navigate to a distant point. Pilots followed railroads or the few roads that existed, or they "dead reckoned," following a compass heading for a calculated time to reach an identifiable point along the way. "See and avoid" was the only method for staying safely away from other airplanes, and that method endures to this day as the last line of defense against mid-air collision in all flight operations. For flights performed using VFR, it remains the primary line of defense.

Sperry’s gyroscopic "artificial horizon” overcame the inability to fly without a visual reference, but flights in the clouds still could not use the visual landmarks to navigate. The visual checkpoints, bonfires, and lighted airways gave way to a new invention - radio - as a means to navigate cross-country. Direction finding antennas were used to "home in" on standard broadcast stations near the cities. A radio facility was developed in 1919 just for aviation called the Adcock or "Four Course" Range, shown in Fig. 1. Its antenna arrangement on the ground created four lines of position emanating from the center on which a steady tone was heard in the airplane. On one side of the course, a Morse code letter "A" was heard (dot-dash); on the other, an " $N$ " (dash-dot). Placing these ground stations so that the course ends joined created radio navigation "airways" that were used to fly from one city to another. Thus the flexibility to fly in and above the clouds was acquired in trade for the flexibility to navigate along any chosen path, now relegated
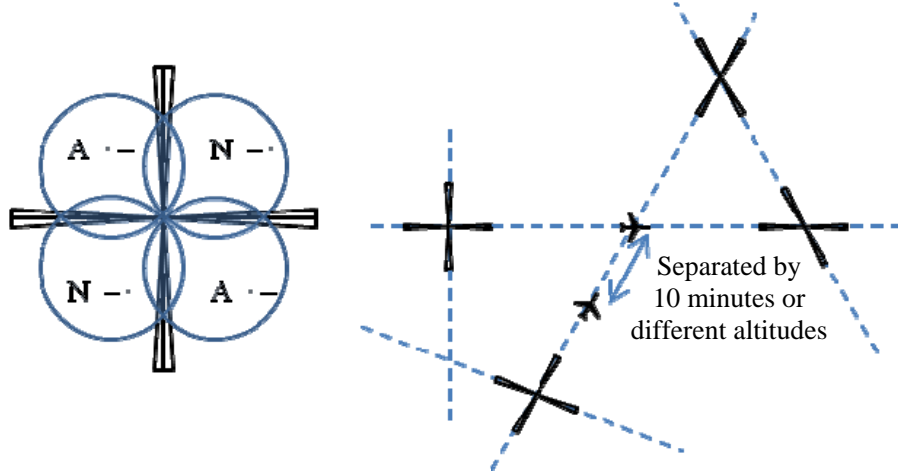

Fig. 1. The Four Course Range provided early ground-based navigation and procedural separation for aircraft operating in and above the clouds. The airways these created placed limits on navigational flexibility. to just those few airways.

Flying in the clouds posed another challenge to early aviators: how to maintain separation from other flights in the clouds. "Air Traffic Control " (ATC), initially used only to adjudicate the use of runways between arriving and departing traffic, was expanded in scope in the mid 1930's to assist in separating en route aircraft. "Procedural" separation was used first, based upon filed flight plans along established airways and flying at a declared altitude. Airplanes at different altitudes or flying on different airways were considered to be "procedurally separated." Where airways crossed, if flights were at the same altitude, they had to cross the intersection at least ten minutes apart. Red and green light guns used in airport traffic patterns were supplemented with teletype communications between control centers and with a company set up by the airlines called Aeronautical Radio Incorporated (ARINC) that had established a radio communications network for air/ground voice messages. Pilot estimates of intersection crossing times were passed through the ARINC operator by teletype to the air traffic control center. Any needed change to the time or altitude of crossing was returned via the same path. Direct radio communication between en route pilots and controllers came many years later and improved the timeliness and reliability of this essentially "one at a time" aircraft separation system. The poor navigational accuracy, surveillance by position report and estimate, and control loop times measured in minutes made inefficient use of the airspace "the norm" and a widely accepted artifact of air traffic control even today. It was the price of all-weather flexibility.

\section{B. Radar Separation}

When radar was introduced to ATC in the 1950s, a new, smaller separation standard was established between the radar-identified aircraft. But the coverage of radar was so limited and the reliability of radar and radio communications so poor that airways and procedural separation were retained in the separation assurance methods throughout the last fifty years, even today. A radar display is shown in Fig. 2. 
Many improvements to radio and satellite navigation took place during the last half century, enabling position determination anywhere and the ability to fly with area navigation (RNAV) along any defined track over land or water, not just between ground stations, with a precision that has shrunk from several miles to a few feet. Theoretically, the coverage, accuracy, and reliability of communications, navigation, and surveillance available today could safely support orders of magnitude increases in traffic density, but those are not the only elements in the air traffic control process for separating aircraft.

The airspace is divided into sectors, within which a radar controller is responsible for providing separation of all aircraft under his/her control. This is accomplished by controllers observing the locations of all such aircraft and projecting their paths forward to check for possible conflicts. Expected conflicts are resolved by issuing an amended clearance to one or both of the affected aircraft.

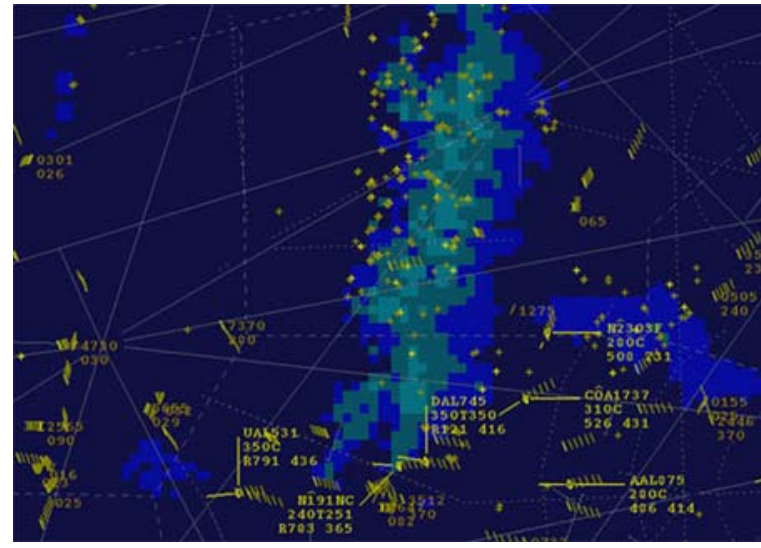

Fig. 2. Radar provided real-time ground-based surveillance and smaller separation between aircraft. However, flexible use of new aircraft navigation capabilities remained limited. Adjustments to heading, route, altitude, or speed are assigned, and the new assignments are executed by the pilots. This process describes the core premise of IFR. The amount of traffic that can be safely accommodated in a single sector is determined by an acceptable cognitive workload for a radar controller. Generally speaking, the fewer the routes, altitudes changes, and speeds of the aircraft, the easier it can be for controllers to predict traffic conflicts and the greater the number of aircraft that can be in the sector at once. Climbing, descending, and maneuvering traffic complicate the conflict detection process and may reduce the number of aircraft that can be safely handled by this method. Thus the ability to operate in the clouds remains in trade for the flexibility to navigate as desired (despite the aircraft's global RNAV capability), and airspace capacity is limited not by the airspace volume, which is indeed vast and could physically support far more traffic (ref. 2), but by the cognitive method of ATC-based separation.

The capabilities of current air traffic control systems require that many restrictive rules and procedures be used to maintain safety. Among these are sector capacity limits, structured routes, altitude stratification, "miles in trail" procedures, speed matching, and cumbersome trajectory change processes. Nevertheless, with all these constraints, the current ATC system does a remarkable job of handling the primary IFR user of the airspace today, airline transportation. It does so using a mix of strategic (procedural), tactical (radar), and pilot (visual) procedures. It also includes a sharing of responsibilities among pilots, controllers, and automation support systems. Controllers will set up a plan for separation, but the pilots must fly that plan for separation to be maintained. Significantly, the IFR process works in all weather conditions, even though it cannot accommodate the traffic densities and operational flexibility that VFR operations enjoy.

\section{The Path Forward - AFR}

\section{A. Recovering Navigational Flexibility}

The previous section reviewed the evolution from visual to procedural to radar-based separation, and the trades that were made by operators to gain all-weather flexibility in exchange for significant limits on navigation flexibility and airspace capacity. The complex IFR-ATC system we have today has its roots in the pilot's original need to rely on ground systems for navigation and separation in non-visual conditions. Imagine how our air traffic system might have evolved differently throughout aviation's first century if the early aviators had all-weather navigation capability and direct access to highly accurate airborne surveillance of their surrounding traffic, regardless of visibility, range, azimuth, or altitude. It raises all sorts of interesting questions on how roles, responsibilities, and even basic procedures might have developed differently. Would controllers be providing traffic separation as one of their primary functions, or would this function have become merged with pilot responsibilities for flight safety, such as terrain and weather avoidance? Assuming the latter case, the interaction between pilots and controllers could have developed quite differently regarding the basic management of aircraft trajectories. Would controllers be authorizing each change to the trajectory, or would pilots have authority and operational flexibility to make "autonomous" changes, even in the clouds?

The historical fact is that these capabilities - all-weather navigation and airborne surveillance - did not exist, and ground-based separation services were the only viable solution of the time. This is no longer the case. Technologies illustrated in Fig. 3 are fundamentally changing the assumptions under which the current separation system operates. 
With Global Navigation Satellite Systems (GNSS), aircraft can precisely navigate to any point on the planet. Automatic Dependent Surveillance Broadcast (ADS-B) technology allows aircraft to directly share their satellite-derived position and avionics-derived intent with other aircraft and ground systems. Aircraft equipped with an ADS-B receiver can know the position and intent of broadcasting traffic nearly as well as the traffic knows itself. Thus, the practical age of allweather navigation and airborne surveillance has arrived. Can we therefore envision a means in which aircraft can regain that flexibility by once again navigating with autonomy and providing their own safe passage with respect to other aircraft, without reliance on ground systems for these purposes? This is the concept of self-separation.

This vision dates back many decades, predating the technology needed to put it into practical use (refs. 3-7). Shortly after World War II, the Radio Corporation of America proposed such a concept to the Civil Aeronautics Authority (CAA) called "Teleran" for TELEvisionRadar Air Navigation." From the mid-1960s to the mid 1970s, considerable research and simulations were performed at the Massachusetts Institute of Technology and the Lincoln Labs using a "cockpit display of traffic information." Operations were proposed

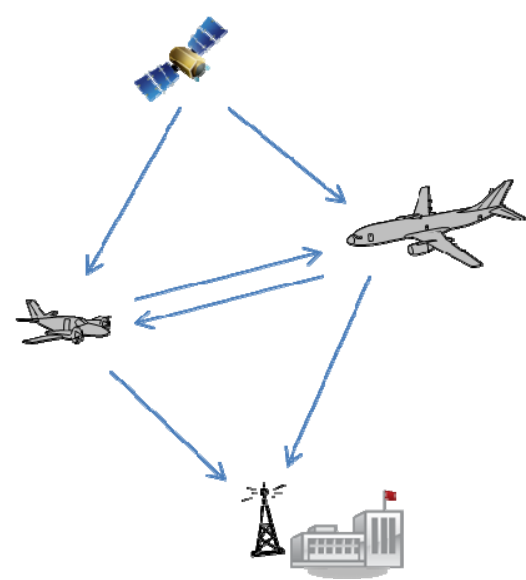

Fig. 3. GNSS and ADS-B technologies enable equipped aircraft to navigate on any desired path and share position and intent with other aircraft and ground systems. using "Electronic Flight Rules," but the enabling technologies simply were not mature enough for practical application. The concept of Autonomous Flight Rules (AFR) is the most recent and detailed vision for aircraft operations that use self-separation (ref. 1). As its name implies, AFR does not propose to replace or redefine VFR or IFR operations, but to become available as a third option for aircraft operators. The rationale for this is discussed in Section IV. AFR also does not negate the necessity of ATC, which remains critical for separating IFR flights and managing high-volume terminal arrivals and departures.

The objective of the AFR concept is to provide an alternative mode of aircraft operations that is safe, efficient, flexible, cost effective, and low risk for aircraft operators. These benefits are discussed further in Section V. Selfseparation is not the objective of AFR operations; rather, it is the primary enabling capability for achieving these benefits. Self-separation enables pilots to safely manage their flights in non-visual conditions in the presence of other traffic. By providing their own separation service, AFR operators remove the burden and responsibility of this function from ATC and gain independence from most of the operational restrictions historically put in place to enable today's ATC system. While AFR operations alone will not change the total number of flights in the airspace, the existence of the capability will provide a mechanism for significant expansion of en-route airspace system capacity, which benefits all airspace users and ATC. It also gains back the operator's flexibility to navigate throughout the airspace, in and above the clouds.

\section{B. The Meaning of "Autonomous" in AFR}

The term "autonomous" can be defined as "not subject to control from outside" or "independent." Even so, an autonomous agent is still considered part of a community subject to rules and constraints. The term was chosen for AFR to elevate two fundamental principles of the proposed operation: the degree of authority the operator has over the trajectory of the aircraft, and the degree of responsibility the operator has to ensure safe operations in a mixed traffic environment. Both principles of this concept are ones of independence. The autonomous authority provides the operator the independence to define and change the trajectory without outside (i.e. ATC) approval, as in VFR, but with additional independence from VFR meteorological and airspace restrictions. The autonomous responsibility compels the operator to independently ensure (without relying on ATC as a ready fallback) that their trajectory does not breach established separation criteria from other traffic, a stronger safety requirement than VFR's "see and avoid." Thus, AFR represents not a "free for all" but rather a structured flight mode with rules and procedures that, while highly flexible, methodically ensures separation safety with the utmost integrity.

\section{Enabled by New Technologies}

One might ask why, if this concept has been around for so long, it has never been given serious consideration for use in regular operations. The answer is twofold, the first having to do with technical capability and the second with the procedural integration of mixed operations. This section addresses the first of those considerations. The second is addressed in Section IV. 


\section{Navigation}

GNSS has provided worldwide RNAV flexibility and precision that could only be dreamed about in decades past. Recently, new flight procedures called "Required Navigation Performance" (RNP) make use of this precision and enable instrument approach and missed approach procedures to be used to much lower weather minima than was previously possible. RNP specifications bound the navigation error budget so that aircraft can be safely flown right next to mountains in Instrument Meteorological Conditions (IMC). RNP also enables closer spacing of procedurally separated routes. AFR requires that the navigation function not just be accurate, but be extremely robust as well. The use of RNP in AFR allows anticipated failure modes to be monitored and backed up, so that losing the on-board capability to fly an intended trajectory near other aircraft is extremely remote. Independent backup navigational capabilities are also now available, such as precision Distance Measuring Equipment or the transmission of groundsensed multilateration back to the aircraft for use in navigation.

\section{Airborne Surveillance}

ADS-B signals will soon prevail in most of the airspace, providing a ready source of surveillance information for airborne systems. ADS-B IN (i.e. receiving capability) is proposed as the primary airborne surveillance system for AFR. While the current Federal Aviation Administration (FAA) rule requiring ADS-B OUT (i.e., transmission only) by 2020 does not include intent information, this may be added to the message in the future. "Flight Object" information on other aircraft trajectories, intended to be available in the future ground automation systems, would aid AFR in the strategic planning portion of the airborne separation system, but safety can be assured by tactical use of the state vector broadcast even in the absence of longer term intent. Just as with the navigation function, however, the airborne surveillance function must be very robust. That means an independent source of the information such as Traffic Information System Broadcast (TIS-B) must be available. The accuracy and latency of TIS-B will drive the larger separation values used by the airborne separation system, in the event of primary system failure. Multilateration could be a suitable source of independent TIS-B data.

\section{Communication Links}

Both the primary and backup means for airborne surveillance depend on data communication to carry out the function. Therefore, both redundant and independent channels to receive the surveillance information must be provided. Separate electrical power, including battery backup may also be required to achieve the necessary robustness of this function. Mode S, Universal Access Transceiver, and other airborne data channels are already in use and are being rapidly supplemented by satellite and terrestrial broadband access to information, so the fulfillment of this requirement is expected to be just an engineering challenge, not an issue of technical feasibility.

\section{Data processing, display and control}

At the time of earlier proposals of this concept, computing power was either very limited or non-existent. What did exist was large, heavy, and very expensive, and it required a lot of human involvement in its operation. Today's automation and robotic software functionalities, computing power, and miniaturization are taken for granted by most of the population, and they truly enable the practical application of self-separation among aircraft. Cockpit systems are now achievable that can robustly perform the conflict management functions (detection, resolution, and prevention) in the presence of other constraints for hazard avoidance and traffic flow management. A sophisticated conflict management prototype system has been developed and tested by airline pilots in simulation over the past decade (refs. 8-10). It is no longer a question of if this can be done, but how to best implement the desired features of the system.

\section{AFR Safety}

Most discussions of air traffic safety contain the argument that a controller must always be responsible for air traffic safety because he/she is the only one with a complete picture of what is going on in the airspace. In fact, controllers rarely have the complete picture, rather only that part of the picture for which they are responsible. The Federal Aviation Regulations are clear that the pilot in command of a flight is the final authority with respect to the operation of the flight and is ultimately responsible for its safety. He/she is expected to see and avoid conflicting traffic even under IFR, because that traffic may not be known to the ATC system. The same is true for weather avoidance. Many weather hazards are unknown to air traffic control, and the pilot is expected to stay clear of those hazards while coordinating the required flight path changes with ATC. In fact, every clearance or instruction issued by ATC is checked by the pilot in command to ensure its compatibility with the safe operation of the aircraft.

The 1958 Federal Aviation Act, which replaced the CAA with the Federal Aviation Agency (FAA), had the intent of improving the safety of airline operations, especially to reduce their use of VFR in the wake of the tragic 
1956 Grand Canyon mid-air collision (ref. 11). The Act was signed into law right at the beginning of the jet age, which marked a dramatic increase in the use of high altitudes and higher speeds. It was not long before the establishment of "positive control" in the upper airspace, in which only IFR flight was permitted. Project Beacon (ref. 11) established the rule requiring the use of transponders in much of the airspace to greatly improve the ability of radar controllers to maintain surveillance in their control sectors. All these changes created the impression that ground-based air traffic control was solely responsible for safety in the skies.

As a practical matter, flight safety is a shared responsibility among all those who have a hand in the flight operation. Pilots and controllers each know a part of the operation and have responsibilities for their part in flight safety. Flight dispatchers and mechanics also see parts of the operation transparent to others and similarly are responsible for those things they control. Separation safety is shared between pilots and controllers because each has information relating to the traffic situation that the other does not.

The safety of any separation system depends on the integrity, accuracy, and speed of the control loop. That loop consists of tracking the aircraft, projecting their trajectories to check for conflict, altering trajectories to resolve the conflict, and checking the result. Traditional ATC does most of the control loop functions on the ground, directed by an air traffic controller using radar, some automation assistance, and voice communication to instruct the pilot to alter the trajectory. The time horizon of the projection for conflicts is on the order of 4 to 8 minutes, depending on workload, airspace design, and other factors. Because this function rests primarily in the ATC system, most of the responsibility lies there as well. The pilot's responsibility consists of altering the trajectory in the directed manner without undue delay.

By contrast, AFR places all the elements of the control loop within the airplane. Automation plays a larger role, doing the tracking, projecting, and conflict detection, directing the corrective maneuver through the flight director or other displays, and checking the result for adequacy. The pilot still follows the guidance and re-directs the aircraft trajectory. The responsibility for safety during the AFR separation process rests with the aircraft operator (for the maintenance of the automated system) and the pilot, as before, for executing the trajectory guidance. The level of safety (i.e., the risk of loss of separation) may be improved with AFR through greater redundancy (both aircraft in conflict detecting the problem and ensuring resolution), speed of execution, and containment of control loop communications completely within the airplane. It is less susceptible to communication failures of all kinds, technical and human, and therefore does not depend on long-term adherence to strategic trajectory planning. The AFR automation will be designed to provide separation from all aircraft in surveillance: AFR, IFR, and VFR. A buffer to account for possibly limited knowledge of IFR intent will increase the separation from IFR aircraft. The buffer also will improve the controller's confidence that the AFR aircraft not under his control is performing the required separation from IFR aircraft under his control. The controller will not be responsible for this separation.

AFR will not enter into service until its safety is assured. Studies and simulations over the past decade have shown the viability of airborne separation logic to safely maintain separation without disrupting the objective of the flight and still meeting other operational constraints (refs. 9, 10, 12-16). The entire system will be tested thoroughly before it is certified to perform the separation function in regular operations. Backups for failures of any component of the system will be designed so that safety can be handled in a tactical manner even in short-notice circumstances.

\section{The Operational Mix}

\section{A. Flight Rules - All for One}

The community of aircraft operators is an aviation brotherhood sharing a common purpose, which is to operate in the airspace with as much flexibility as possible to achieve their individual missions and goals. Assuredly, their missions and goals are as diverse as imaginable, even as our imagination for the utility of flight continues to expand (e.g. unmanned vehicles). From the earliest days of flight to today, many operators seek simply to gain perspective on the world and the atmosphere above it. Sightseeing, aerial photography, reconnaissance, industrial inspections, search and rescue, atmospheric sampling - these missions typically begin and end at the same airport and often have no predetermined timing or even trajectory. The objective is to take off, fly for a while, accomplish a particular objective, and then decide where to go next before returning home. Training flights have similar characteristics. Other missions such as crop dusting and fire fighting provide uniquely valuable access to our planet from above, and although the target location is known before the flight, the plan to get there may be as changeable as the wind. Such flights reap the benefits of VFR - our currently most flexible mode of airspace access. Few operational restrictions greet a VFR pilot, and thus it remains the preferred mode for many operators on round-robin missions.

Many operators, of course, fly aircraft to gain transport from point to point. They generally do so because the distance is too long or treacherous to be efficiently achieved with alternative transportation, because time is of the essence (e.g. business deadlines), or because they have customers willing to pay them for reliable and timely air 
transport (either for themselves or their packages or cargo). For these aviators, IFR flight provides definite advantages. Foremost among these is independence from Visual Meteorological Conditions (VMC). When time is of the essence or long distances must be traveled, the likelihood that VMC will prevail throughout the flight cannot be trusted, and IFR flight becomes a practical or business necessity. For many other operators, improved safety is the \#1 reason to file IFR. Having another person watching out for your separation from other aircraft is often worth the price of admission, particularly where airspeeds are high or ability to see traffic coming is otherwise uncertain. And in today's evolved airspace system, IFR flight brings additional advantages, such as permission to operate in the Flight Levels and flow-managed arrival to high-demand airports. The latter is a significant benefit in pilot workload, to which anyone who has attended a fly-in can attest.

The introduction of AFR will complement VFR and IFR, not replace either one. All three would exist for the benefit of the operator (all for one), and the choice of flight rules will be up to the operator for each flight, according to where they fly, their mission, and their business model. Neither VFR nor IFR offers as much operational flexibility as will AFR, which effectively combines the weather independence of VFR with the safety and airspace access of IFR, but without most of the restrictions that historically emerged to enable the ATC system to function. AFR will be well suited for both round robin and cross-country (point-to-point) transport missions. However, given the vast diversity of missions and operators, all three flight modes would likely be heavily demanded well into the future.

\section{B. AFR and Airspace - One for All}

AFR is for anyone (one for all) - air carriers, air taxis, private and commercial General Aviation (GA) operators, corporate aircraft, military airspace users, and unmanned aerial vehicles. Any operator can elect to fly AFR, provided they invest in the required equipment and training, and adhere to the rules (just like VFR and IFR). The mission and economics of each flight will determine whether to file VFR, IFR, or AFR. In fact, composite flight plans could even be filed, as today, where a given flight can be conducted under multiple flight rules along the route. A likely candidate would be a flight to or from a high-demand airport, where terminal IFR services may be worth the restrictions of ATC but en-route services may not. Since all three flight modes are intended to serve the interests of the airspace user, significant flexibility for users to select the most appropriate mode for each flight is paramount.

Equally important to the choice of AFR, IFR, or VFR is the value to operators of mixing these operations in shared airspace (one - airspace - for all), as shown in Fig. 4. Indeed, it is a significant detriment to operators when one operational mode is segregated from another. Sometimes called "separating from airspace," segregation of operations is an important element enabling today's ATC system. The restrictions take many forms. VFR flights are restricted from the Flight Levels, must fly in special corridors through some terminal airspace, and give up significant maneuvering authority when inside Class B and C airspace, all because of the presence of IFR traffic. IFR flights, though not heavily restricted by VFR traffic, are also subject to segregated practices. They are generally asked to remain on airways (and typically on specific airways known as ATC "preferential routes"), are often restricted from uninterrupted climbs, must follow hemispheric rules at altitude, may be held slow or delayed to accommodate sector capacity restrictions, are often rerouted en masse around weather, and are subjected to early step-downs in the arrival. Fortunately, the segregation between VFR and IFR operations is minimal, relegated mostly to those areas where large differences in cruise speed make ATC a sensible intermediary.

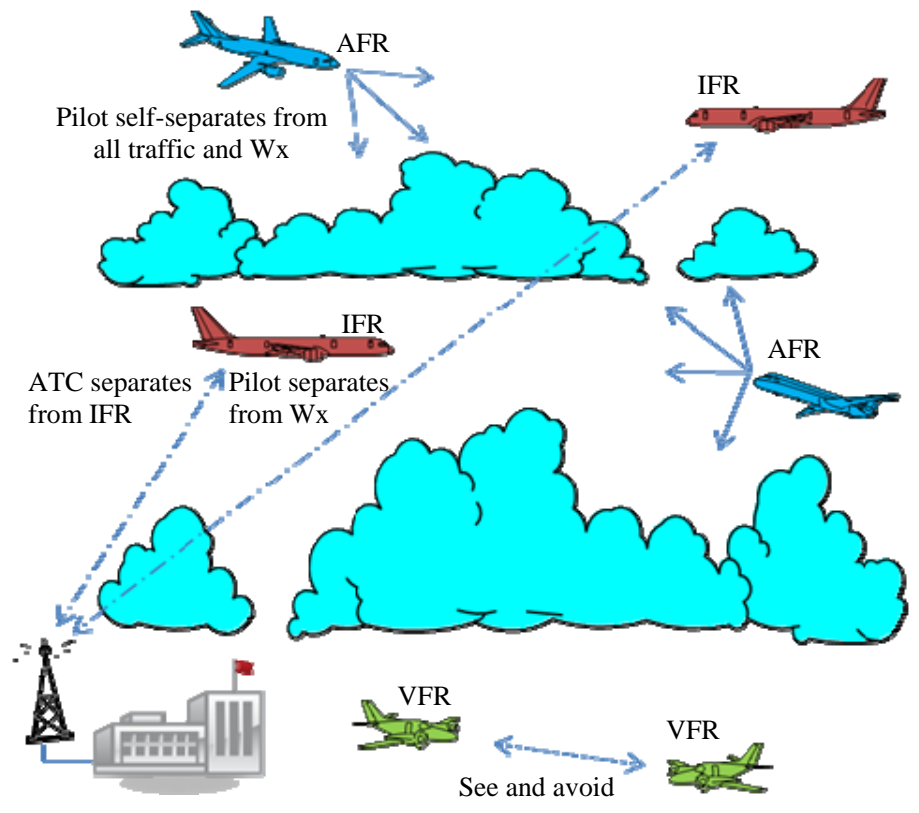

Fig. 3. An integrated mix of AFR, IFR, and VFR operations in shared airspace provides the flexibility for airspace users to safely and efficiently meet diverse missions and goals. 
Minimizing the segregation between all three flight modes - AFR, IFR, and VFR - will be critical to their mutual ability to serve the user community effectively.

As the new operation, it will be incumbent on AFR operations to take on the burden of facilitating the successful integration with the other modes - in particular, IFR and the existing ATC system. For this reason, the AFR concept of operations was designed to facilitate mixed operations from the outset. The concept specifies that IFR aircraft will have the right-of-way in all traffic conflicts with AFR aircraft, and that AFR aircraft will assume the burden of resolving these conflicts by maneuvering clear. Furthermore, the air traffic controller will not be responsible for monitoring the AFR flight or for conflicts between AFR and IFR aircraft. These stipulations are intended to make mixed operations occur naturally and without disruption to the complex and intricate IFR-ATC system we have available today and cannot afford to redesign. The burden taken on by AFR will be more than offset by the breadth of benefits these operators will receive (Section V).

\section{Why New Flight Rules?}

The eventual emergence of AFR operational capabilities could be considered a foregone conclusion, if one takes the larger historical view. As discussed in Section II, before separation services existed, there was only "see and avoid," a fully distributed, airborne function that continues to this day in VMC. As operations moved into the clouds, where neither visual navigation nor "see and avoid" were possible, ground-based systems emerged to support both navigation and surveillance as the only alternative. Communications were introduced to send intent (e.g. next waypoint estimated crossing time) to the ground and separation clearances to the aircraft. Today, technology has progressed to the point where aircraft can navigate without ground-based aids and can communicate position and intent with nearby aircraft in real time. In fact, navigation precision is now greater and surveillance information more accurate and timely than was available to ATC through much of its history. In addition, onboard computational power and software capabilities now exist to rapidly process this information and provide highly efficient, automated, and reliable conflict detection, resolution, and prevention services, not to mention unprecedented flight optimization (ref. 8). In essence, aircraft have acquired and significantly exceeded all that was lacking in the early days of IMC flight that required operators to rely on ground-based services for separation. One can see how AFR emergence would be the natural outcome of this new reality. One might ask, then, in the spirit of technological revolution, whether self-separation should simply replace IFR, and for that matter VFR, as the way we would have operated all along had the technology been available. In other words, why propose a new set of rules, AFR? Why not just propose an update to the existing IFR and VFR rules?

There are at least four reasons that justify the new rule set. The first is to preserve what works. While it is true that ground-based separation services were created to compensate for a lack of airborne information and capability that may soon no longer be lacking, IFR and ATC have evolved over decades into a highly sophisticated, productive, and safe system that fuels much of the current aviation economy and perfectly meets the needs of many aircraft operators. To disrupt this system would impact many operators who use it every day and are completely satisfied. In addition, the IFR-ATC system is highly complex and intricate, with webs of interdependence across rules, procedures, technologies, information flows, responsibilities, pilot/controller training, and regulations. Introducing a fundamental change could easily upset this balance and have significant undesired repercussions throughout the system. In fact, this is the massive challenge facing the FAA's Next Generation Air Transportation System (NextGen). The far simpler and much more sensible approach is to leave what works alone and introduce the new capabilities in a parallel and non-interfering manner.

Introducing a new rule set also provides the flexibility to define it properly. As a community, we will be able to craft just the right regulatory policies, performance standards, certification requirements, training programs, etc. to specifically suit the new operation. The alternative - combing through all of the IFR material and rewriting it broadly enough to encompass both modes of operating (if even possible) - would almost certainly compromise both the current IFR-ATC system and the future promise of AFR. Separate rule sets will allow each to be tailored to their operation for optimal performance, and they will permit far easier adjustments to each as they independently mature over time.

Phasing the new operation into service will also be made much easier by a new rule set. With the IFR-ATC system uninterrupted and virtually unchanged, AFR operations can be introduced gradually and safely, one aircraft at a time. Even the first equipped aircraft and authorized crew will be able to operate in AFR, rather than wait for a critical mass of operators to equip and participate. It will gain immediate benefits without removing benefits from the other flights or otherwise disrupting their operations. The flight may even revert to IFR without burdening the ATC system, because that same flight would have otherwise been filed as IFR under ATC control. With this gradual implementation of AFR, if early experience dictates that adjustments need to be made to AFR policies, this 
can be done without the massive disruption that revising IFR policies would cause. New rules for just the new operation enable this.

Finally, new flight rules are necessary to set proper expectations for pilots and controllers, but mostly pilots. The act of choosing the set of flight rules under which you will operate your flight is a public declaration of responsibility, particularly in the areas of flight visibility and traffic separation. By conducting a VFR flight, the pilot declares responsibility for remaining in VMC and therefore also for separation, or more specifically see-andavoid. By filing an IFR flight plan, the pilot effectively transfers responsibility for separation to ATC in return for authorization to operate in IMC. However, if the IFR pilot declares a traffic aircraft in sight, ATC can delegate separation responsibility back to the pilot for that specific encounter. While it makes practical sense to do so, this unscripted and sometimes temporary shifting of responsibility during the flight can easily blur the lines of separation responsibility. This is particularly true if the pilot later loses sight of the traffic. Detailed rules and procedures have been developed to handle this contingency, but nevertheless, the introduction of self-separation in IFR would only amplify the potential for such confusion. It is far safer for separation responsibility to be an informed and premeditated decision by the pilot and to be publically declared. Filing an AFR flight plan will make clear the pilot's intent to self-separate, and it will set the pilots' expectation that, barring a true emergency, this responsibility will endure throughout the flight.

\section{Anticipated Benefits}

AFR flight, enabled by the self-separation capability, should not be pursued just because it is technically feasible. It is the very substantial benefits brought about by these operations that provide the strong incentive to move ahead with this capability. The benefits to the operators who take advantage of this option can be extraordinary, but there are benefits to the ATC service provider and other operators still using IFR and VFR as well. This section lists and explains those benefit types and mechanisms.

\section{A. Improve Safety}

The overall risk of mid-air collision is expected to be reduced by providing lower risk to individual flights through their use of self-separation equipment and procedures, and by reducing the number of aircraft within the ground-based separation management system. This risk reduction is enabled by automatic monitoring of the traffic situation on multiple aircraft (i.e. the redundancy provided by distribution, with the equipment on two or more AFR aircraft monitoring the same encounter) and by locating the entire control loop within the cockpit so that control loop time is reduced and vulnerability to air-ground communication failures is eliminated. As the ground ATM system becomes responsible for fewer aircraft, the service to those remaining aircraft would improve, and the risk of failures would also correspondingly decrease. Safety of separation from other hazards such as severe weather is also improved by virtue of both functions being located within the aircraft.

\section{B. Improve Efficiency and Reduce Delay}

IFR aircraft are routinely delayed by congestion at terminal departure fixes and in en-route airspace. AFR aircraft ready for departure would be eligible to receive a departure clearance without these delays. The departure approval would be enabled by the AFR aircraft assuming the burden for separation responsibility and thus not contributing to ATC-related "congestion" that triggered the ground delay program. AFR aircraft might similarly be exempt from common "miles-in-trail” restrictions over IFR departure fixes.

While en route, AFR flights using self-separation equipment and procedures would be able to execute their business trajectories more efficiently because their operation would be independent of ATC constraints driven by the quantity of ground-managed IFR aircraft, such as sector load balancing and the longer lead times needed to approve requests. The improved flight efficiency would be measurable as reduced block time and fuel burn, as well as improved on-time performance.

AFR operations would have greater throughput in regions of convective weather because individual weather rerouting decisions would be made per-aircraft, rather than the "playbook" decisions applied to large groups of aircraft that can significantly impede throughput and lengthen weather deviation routes. AFR consolidates decisionmaking for traffic separation and weather avoidance decision-making from two places (ATC for traffic, flight deck for weather) to one (flight deck for both), thereby allowing more efficient use of the weather-impacted airspace and a greater number of viable routing and altitude options. 


\section{Increase Flexibility}

Self-separation is designed to maximize the flexibility afforded to AFR flights by eliminating the requirements for pre-approval of trajectory changes and conformance to most static route and airspace constraints. AFR aircraft could therefore change their trajectory as frequently as conditions warrant and allow, without imposing a burden on ATC or other aircraft. This added flexibility would also be a major contributor to flight efficiency, being advantageous not only in choosing an initial business trajectory during flight planning, but also as changing conditions en-route become apparent to the pilots. For airline and other flights involved in air transportation, this includes altered winds, turbulence, actual locations of severe weather, and changing company business objectives. Any of these dynamic factors translate to a different optimum flight profile - vertically, laterally, and in speed toward destination. The increased flexibility should also be beneficial when weather delays are anticipated at the destination airport. Working with ATC's Traffic Flow Management system, AFR operators would negotiate a scheduled time of arrival consistent with other traffic and the latest weather forecast, and they would negotiate a self-selected departure time to minimize the probability of en-route holding or diversions and thus the excess fuel carried onboard for these purposes.

\section{Lower Costs}

AFR equipment costs to users are expected to be rapidly offset by operational cost savings from the increased flight efficiency and flexibility (ref. 17). Furthermore, these benefits would be immediately available even to the first operator to use AFR, as a result of AFR being a per-aircraft application. Aircraft fleet operators could select which aircraft types and routes would benefit the most from AFR and thereby reduce total investment costs across the fleet by not equipping all aircraft or training all flight crews for AFR. The return-on-investment period of AFR capability is expected to be significantly shorter than those dependent on time-consuming implementation of extensive ground-based infrastructure, a process not under aircraft operator control. Once the planned surveillance systems are in place, there would be no need to coordinate the implementation timing of airborne equipment purchases with ground system operational readiness. Similarly, future upgrades to AFR capability might be put in service faster than upgrades to extensive ground-based infrastructure, thus accelerating the return-on-investment period.

Much of the equipment required for AFR operations is either in service or emerging independently of the AFR concept. Most airline aircraft and many GA and military aircraft are already equipped with Flight Management Systems and multi-function displays, and these numbers are rapidly increasing. ADS-B OUT-capable aircraft are also increasing rapidly in number, and the current rule requiring this capability by 2020 will ensure eventual ubiquitous coverage of airborne surveillance in the most heavily traveled airspace. The additional cost for ADS-B IN, receipt of other's intent data from airborne or ground sources, and the flight-deck automation logic for selfseparation are expected to be small compared to the operational savings. For many aircraft, ADS-B IN equipment and processing capability will have already been installed to enable one or more of the many nearer-term ADS-B applications such as Interval Management currently under development.

Ground system costs should also be positively affected by AFR. Performing separation from the ground requires iterative processing of $n^{2}$ traffic interactions, where " $\mathrm{n}$ " is the number of aircraft under ground control. In contrast, the distributed processing enabled by AFR increases only linearly with increasing numbers of aircraft. Selfseparation could therefore reduce complexity in communications and automation systems that robustly handle the $\mathrm{n}^{2}$ problem, especially for an order of magnitude increase in traffic as might come from widespread use of unmanned aircraft, and in local regions not well covered by ATC communications. It does so by reducing the overall traffic growth that must be managed by ground systems. Since the ground system is experiencing frequent saturation today, AFR operations could prevent a further increase in operator and system costs associated with travel delays and recovery that would otherwise accrue as traffic demand from both manned and unmanned aircraft continues to grow in the future. Ground system costs may even decrease, depending on the number of aircraft remaining in the IFR system.

\section{E. Reduce Implementation Risk}

AFR would provide the aforementioned benefits without dependence on government implementation of a system-wide, ground-based, automated separation infrastructure, while remaining compatible if and when such a system is implemented. Other than the required policy and regulatory changes to establish AFR and the provision of information from government sources (e.g. surveillance of non-broadcasting aircraft), the development and implementation schedule of AFR is determined by the operator community. Once the policy and regulatory changes are in place, the first aircraft equipped and authorized for AFR may use the procedures and gain immediate benefits without waiting for a sizable population to equip. These factors, which allow earlier yet gradual introduction of AFR 
operations into the airspace, could overcome the most challenging obstacle to achieving NextGen's anticipated benefits: the transition period.

\section{Conclusion}

Aviation visionaries have known for decades that to obtain the full potential of our vast airspace, it would be necessary to use means other than the historically-evolved methods of air traffic control to provide separation between aircraft. To achieve true navigational and operational flexibility in all-weather conditions, conflict management would need to be performed locally, automatically, and reliably, while maximizing adherence to flight objectives and minimizing perturbations to other flights. Self-separation is the capability that enables these requirements to be met, provided it is technically achievable and made operationally acceptable. The concept of AFR, described in detail in Ref. 1, is designed to meet these twin objectives.

The ability for aircraft to separate themselves has been elusive since aircraft started routinely entering the clouds and depending on ground-based services for navigation and separation. Now, with the emergence of new technologies, self-separation is again within reach as a practical operation. Highly accurate global navigation, air-toair surveillance, and onboard data processing are available or will be entering operational use within the decade. With engagement and encouragement by the user community, self-separation avionics can be created, tested, and approved in the same period of time. Giving IFR flights the right-of-way paves the way for a smooth integration of AFR flights into the current IFR/VFR mix. AFR participation in airport Traffic Flow Management initiatives allows control to smoothly revert to ATC in the traffic patterns and busy terminal airspace.

AFR is designed to complement IFR and VFR as a third option available to all airspace users. Operational benefits would accrue to AFR flights, including the very first AFR flight, in the form of delays not taken and the flexibility to optimize the business trajectory and autonomously re-plan as conditions warrant. In addition, by using AFR, many GA operations would gain access to significantly more airspace in IMC while maintaining the flexibility of VFR. As more operators choose AFR, the correspondingly fewer number of IFR flights would relieve the pressure from the ATC system, enabling greater flight efficiency for all. As economic conditions and new aircraft capabilities add more flights into the airspace in the future, scalable AFR operations could accommodate the increase without requiring additional ground-based infrastructure to manage the load.

Key hurdles to clear on the path to approved AFR use are the design, testing, and certification of airborne equipment for self-separation and the creation of rules and procedures for its use. The aviation user community must provide the driving force to see these equipment, rules, and procedures through the government processes that establish them. While AFR is compatible with NextGen plans for modernizing ground-based traffic management, selfseparation in mixed airspace is not in the FAA's current concept of operations. It will take a strong push from the airspace users to change that. However, once in place, the AFR option will break the locks of historically derived airspace restrictions, paving the way to flight efficiencies and all-weather flexibility never before available, allowing all who would use the airspace to experience truly spacious skies.

\section{References}

\footnotetext{
${ }^{1}$ Wing, D.J. and Cotton, W.B., Autonomous Flight Rules: A Concept for Self Separation in US Domestic Airspace, NASA Technical Paper (to be published), 2011.

${ }^{2}$ Hasan, S., Leiden, K., Mondoloni, S., Kozarsky, D., and Green, S., “An Initial Benefits Assessment of Distributed Air/Ground Traffic Management Concept Elements, AIAA-2003-6806, 2003.

${ }^{3}$ Francis, D. "Safety in the Soup: Automatic Landings Now, "Teleran” Soon, Assure All-Weather Service on the Airlines," Popular Science, Vol. 148, No. 3, pp74-77, March 1946.

${ }^{4}$ Cotton, W.B., New Directions in Air Traffic Control at Kennedy Airport, Master's Thesis, Course XVI, M.I.T., August 1965.

${ }^{5}$ Cotton, W.B., Formulation of the Air Traffic System as a Management Problem, IEEE Transactions on Communications, January, 1973

${ }^{6}$ Connelly, M.E., Simulation Studies of Airborne Traffic Situation Display Applications - Final Report, Electronic Systems Laboratory, M.I.T., May, 1977.

${ }^{7}$ Andrews, J.W. and Hollister, W.M., Electronic Flight rules: An Alternative Separation Assurance Concept. Project Report ATC-93, Lincoln Laboratory, Massachusetts Institute of Technology, 31 December 1980.

${ }^{8}$ Ballin, M., Sharma, V., Vivona, R., Johnson, E., Ramiscal, E., A Flight Deck Decision Support Tool for Autonomous Airborne Operations. AIAA-2002-4554, 2002.

${ }^{9}$ Barhydt, R. and Kopardekar, P., Joint NASA Ames/Langley Experimental Evaluation of Integrated Air/Ground Operations for En Route Free Maneuvering. 6th USA / Europe Air Traffic Management R\&D Seminar, Baltimore, MD, 2005.
} 
${ }^{10}$ Wing, D.J., Prevot, T., Murdoch, J.L., et al, Comparison of Ground-Based and Airborne Function Allocation Concepts for NextGen Using Human-In-The-Loop Simulations, AIAA-2010-9293, 2010.

${ }^{11}$ Nolan, M.S., Fundamentals of Air Traffic Control, $3^{\text {rd }}$ ed., Brooks/Cole, Pacific Grove, CA, 1999, Chap 1.

${ }^{12}$ Hoekstra, J. M., Designing for Safety the Free Flight Air Traffic Management Concept, National Aerospace Laboratory NLR-TP-2001-313, Amsterdam, The Netherlands, 2001.

${ }^{13}$ Consiglio, M.; Hoadley, S.; Wing, D.; and Baxley, B.: Safety Performance of Airborne Separation: Preliminary Baseline Testing. AIAA-2007-7739, 2007.

${ }^{14}$ Blom, H.A.P., Obbink, B.K., Bakker, G.J., Safety Risk Simulation of an Airborne Self-Separation Concept of Operation, AIAA-2007-7729, 2007.

${ }^{15}$ Consiglio, M.; Hoadley, S.; Wing, D.; Baxley, B.; and Allen, B.: Impact of Pilot Delay and Non-Responsiveness on the Safety Performance of Airborne Separation. AIAA-2008-8882, 2008.

${ }^{16}$ Muñoz, C., Butler, R., Narkawicz, A., Maddalon, J., and Hagen, G., A Criteria Standard for Conflict Resolution: A Vision for Guaranteeing the Safety of Self-Separation in NextGen, NASA/TM-2010-216862, 2010.

${ }^{17}$ Stouffer, V. and Hasan, S., Initial Life-Cycle Cost/Benefit Assessments of Distributed Air/Ground Traffic Management Concept Elements, AIAA-2004-6452, 2004. 